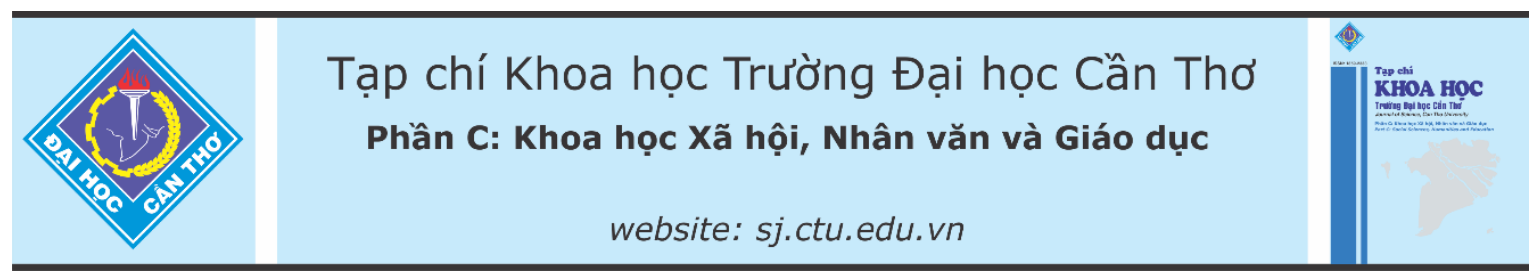

DOI:10.22144/ctu.jvn.2021.054

\title{
CÔNG TÁC CỐ VẦn HỌC TẬP CÁC NGÀNH KỸ THUẬT TẠI KHOA CÔNG NGHỆ - TRƯỜNG ĐẠI HỌC CẦN THO
}

\author{
Nguyễn Thị Thuận và Nguyễn Văn Cương* \\ Khoa Công nghệ, Truờng Đại hoc Cần Tho \\ *Nguoòi chịu trách nhiệm về bài viết: Nguyễn Văn Cuoong (email: nvcuong@ctu.edu.vn)
}

\section{Thông tin chung:}

Ngày nhận bài: 08/11/2020

Ngày nhận bài sưa: 07/01/2021

Ngày duyệt đăng: 28/04/2021

\section{Title:}

Management of academic advising in engineering programs at College of Engineering

Technology - Can Tho University

\section{Tù khóa:}

Công tác cố vấn học tập, cố vấn học tập các ngành kỹ thuạt, quản lý công tác cố vấn học tập, tăng cường công tác cố vấn học tập

\section{Keywords:}

Academic advising, academic advising in engineering programs, management of academic advising, strengthening the academic advising

\begin{abstract}
This study is aimed to investigate and evaluate the current status of academic advising in engineering programs at College of Engineering Technology - Can Tho University. The analysis results are based on the feedbacks of 66 lecturers who are performing the task of academic advisors in 119 speciallized classes of training programs in engineering sector. The analyzed contents are focused on qualities and criteria of academic advisor or counselor, the current state of academic advising activities, the advantages, and disadvantages that can affect academic advising activities. Then, some suggestions are made to improve and to strengthen the academic advising in the coming years.
\end{abstract}

\section{TÓM TÁT}

Nghiên cúu này nhằm tìm hiểu và đánh giá thực trạng của công tác cố vấn hoc tập trong các chuoong trình kỹ thuật của Khoa Công nghệ Truờng Đại học Cần Tho: Kết quả phân tích dựa trên ý kiến phản hồi của 66 giảng viên đang thưc hiện nhiệm vu cố vấn học tập trong 119 lớp chuyên ngành thuộc các chuoong trình đào tạo của khối ngành kỹ thuật. Nội dung phân tích tập trung vào chất luợng và tiêu chuẩn của cố vấn học tập, thực trạng của hoạt động cố vấn học tập, nhũng thuận lợ và khó khăn có thể ảnh hưởng đển hoạt động cố vấn học tập. Tù đó đura ra một số đề xuất nhằm cải thiện và tăng cuờng công tác cố vấn hoc tập trong nhũng năm tiếp theo.

\section{GIỚI THIỆU}

Phương thức đào tạo theo học chế tín chỉ là một loại hình đào tạo có nhiều ưu điểm và mang lại hiệu quả cao, ra đời và được áp dụng đầu tiên vào năm 1872 tại Trường Đại học Harvard của Mỹ. Từ đó, mô hình này được phát triển rộng khắp trên thế giới và được coi là mô hình đào tạo tiên tiến nhất cho đến nay. Phương thức đào tạo theo học chế tín chỉ đòi hỏi phải có hệ thống cơ sở vật chất và kỹ thuật tốt, cùng với đội ngũ giảng viên có trình độ chuyên môn, nghiệp vụ cao; trong đó không thể bỏ qua vai trò của cố vấn học tập (CVHT). CVHT có vai trò và vị trí đặc biệt quan trọng trong đào tạo tín chỉ, là người có ảnh hưởng trực tiếp đến chất lượng học tập và sự thành công trong học tập, rèn luyện của sinh viên trong quá trình học tập (Nguyễn Nguyệt Minh, 2015). Mỗi CVHT là một mắt xích then chốt trong mối quan hệ: nhà trường (giảng viên, chương trình đào tạo, nội quy, quy định, các hoạt động,...) - sinh viên - thị trường lao động. CVHT là một chuyên gia tư vấn về chương trình học, kế hoạch học tập, thị trường việc làm cho sinh viên, đồng hành cùng sinh viên trong suốt quá trình học. CVHT cũng là người 
định hướng, tư vấn, giám sát hoạt động học tập và rèn luyện của sinh viên, giúp sinh viên nhận thức được tầm quan trọng của quy chế, chương trình đào tạo (CTĐT), kế hoạch học tập, phương pháp học tập; để từ đó sinh viên tự xây dựng cho mình một chương trình, một kế hoạch, một phương pháp học tập hiệu quả nhất đạt được mục tiêu học tập phù hợp với năng lực và hoàn cảnh cá nhân. Nói cách khác, nhiệm vụ của cố vấn học tập là giúp cho quá trình cá nhân hóa học tập của sinh viên được diễn ra một cách tốt nhất (Trần Thị Minh Đức \& Kiều Anh Tuấn, 2012).

Trường Đại học Cần Thơ đã bắt đầu áp dụng phương thức đào tạo theo học chế tín chỉ từ năm 1995, tuy nhiên đến năm học 2007 - 2008 thì mới được áp dụng một cách đầy đủ và triệt để. Hơn 12 năm áp dụng phương thức đào tạo theo học chế tín chỉ cho thấy CVHT có vai trò đặc biệt quan trọng và không thể thiếu (Trường Đại học Cần Thơ, 2011). Theo quy định về công tác học vụ dành cho sinh viên trình độ đại học hệ chính quy của Trường Đại học Cần Thơ, lớp chuyên ngành là lớp được hình thành từ đầu khóa học cho đến cuối khóa học. Tổ chức lớp chuyên ngành để duy trì các hoạt động đoàn thể, lao động, xét kết quả rèn luyện, xét khen thưởng, phổ biến những thông tin của Trường, khoa đến sinh viên. Mỗi lớp chuyên ngành có mã số riêng và được bố trí một CVHT để giúp đỡ sinh viên (Trường Đại học Cần Thơ, 2020a). Năm 2020, Trường Đại học Cần Thơ đã ban hành quy định về công tác cố vấn học tập, trong đó quy định rõ 8 nhiệm vụ của CVHT (Trường Đại học Cần Thơ, 2020b). Các nhiệm vụ CVHT được quy định gồm: (1) học tập nghiên cứu nắm vững $\mathrm{CTĐT}$, quy định công tác $\mathrm{CVHT}$, chế độ chính sách đối với sinh viên để có thể tư vấn, hỗ trợ giúp đỡ cho sinh viên trong quá trình học tập, rèn luyện; (2) nắm danh sách lớp, thông tin cá nhân của sinh viên; (3) tư vấn cho sinh viên xây dựng kế hoạch học tập toàn khóa; hướng dẫn sinh viên đăng ký học phần ở từng học kỳ để hoàn thành kế hoạch học tập; tham gia nghiên cứu khoa học và tham gia các hoạt động học thuật khác; (4) tổ chức họp lớp thực hiện đánh giá kết quả rèn luyện trực tuyến; theo dõi kết quả học tập của từng sinh viên ở mỗi học kỳ; (5) nắm tình hình lớp mình phụ trách, tiếp nhận, xử lý hoặc tư vấn cho sinh viên trong quá trình học tập, rèn luyện, và các vấn đề khác có liên quan; (6) tham dự họp và tư vấn cho Trưởng khoa trong công tác khen thưởng, kỷ luật, xét tốt nghiệp, xét chọn học bổng; (7) phối hợp với các đơn vị, cá nhân có trách nhiệm xử lý các vấn đề liên quan đến sinh viên do lớp mình cố vấn; (8) ghi nhận tình hình sinh viên và báo cáo cho Trưởng khoa hoặc lãnh đạo khoa phụ trách.
Khoa Công Nghệ hiện đang quản lý 14 ngành/chuyên ngành đào tạo bậc đại học, với tổng số sinh viên theo học trên 6.500 sinh viên. Hiện nay, trung bình trong mỗi học kỳ có 4 buổi họp CVHT với sinh viên được xếp cố định theo kế hoạch thời khóa biểu học tập của sinh viên. Trong các buổi họp này, ngoài việc nắm tình hình về tâm tư, tình cảm hoặc các kiến nghị của sinh viên; CVHT còn phải phổ biến hướng dẫn cho sinh viên về CTĐT, cách xây dựng kế hoạch học tập, các thông tin của Trường/Khoa, định hướng và tư vấn nghề nghiệp cho sinh viên. Bên cạnh các buổi họp CVHT trực tiếp, CVHT còn sử dụng các kênh thông tin khác thông qua điện thoại, email, group trên mạng xã hội để trao đổi và tư vấn cho sinh viên khi cần thiết.

Bài viết này trình bày kết quả khảo sát và đánh giá thực trạng các vấn đề liên quan đến công tác CVHT các ngành kỹ thuật như: tiêu chí lựa chọn và công việc của CVHT, các hoạt động liên quan đến công tác CVHT, thuận lợi và khó khăn của công tác CVHT; từ đó đưa ra các đề xuất nhằm cải thiện và tăng cường công tác quản lý CVHT ở Khoa Công Nghệ - Trường Đại học Cần Thơ.

\section{PHƯƠNG PHÁP NGHIÊN CÚU}

Công tác liên quan đến CVHT được khảo sát với 66 giảng viên ( 25 tiến sĩ, 41 thạc sĩ) đang làm nhiệm vụ CVHT đối với 119 lớp chuyên ngành của các ngành/chuyên ngành kỹ thuật thuộc Khoa Công Nghệ. Trong đó, có 12 CVHT nữ, chiếm tỷ lệ $18 \%$; 54 CVHT nam, chiếm tỷ lệ $82 \%$. Có 23 giảng viên $(35 \%)$ có thời gian công tác từ 10 đến 15 năm; 43 giảng viên $(65 \%)$ có thời gian công tác trên 15 năm; không có giảng viên nào có thời gian công tác dưới 10 năm. Đặc biệt, có 06 giảng viên $(9 \%)$ lần đầu tiên làm công tác CVHT.

Nội dung bảng câu hỏi khảo sát tập trung vào các vấn đề chủ yếu sau:

a.Những vai trò và đặc điểm, kinh nghiệm mà CVHT cần có;

b.Các nội dung cần sinh hoạt với sinh viên trong các buổi họp CVHT;

c.Phương thức triển khai các công tác quản lý sinh viên (trao đổi và thu thập thông tin) của CVHT;

d.Những thuận lợi - khó khăn trong việc triển khai thực hiện công tác CVHT;

e.Những giải pháp đề xuất của giảng viên để tăng cường công tác CVHT. 
Bảng 1. Số lượng CVHT tham gia khảo sát

\begin{tabular}{clcc}
\hline TT & Ngành học & Số luợng $\boldsymbol{C V H T}$ & Só́ lớp chuyên ngành \\
\hline 1 & Kỹ thuật cơ khí (3 chuyên ngành) & 11 & 24 \\
2 & Kỹ thuật Cơ điện tử & 5 & 11 \\
3 & Kỹ thuật điều khiển \& tự động hóa & 7 & 19 \\
4 & Kỹ thuật Máy tính & 8 & 11 \\
5 & Kỹ thuật điện tử - viễn thông & 4 & 6 \\
6 & Quản lý Công nghiệp & 3 & 6 \\
7 & Kỹ thuật điện & 8 & 14 \\
8 & Kỹ thuật xây dựng & 6 & 8 \\
9 & Kỹ thuật xây dựng Công trình giao thông & 3 & 4 \\
10 & Kỹ thuật xây dựng Công trình thủy & 3 & 4 \\
11 & Công nghệ KT Hóa học \& KT vật liệu & 8 & 12 \\
\hline & Tồng cộng & 66 & 119 \\
\hline
\end{tabular}

Phương pháp thu thập thông tin được thực hiện trực tuyến (online) bằng công cụ "Google form", các thông tin phản hồi từ CVHT sẽ được thu thập, thống kê và phân tích bằng phần mềm Excel.

\section{KẾT QUẢ VÀ THẢO LUẬN}

\section{1. Đặc điểm, kinh nghiệm cần có của CVHT}

CVHT phải là người có trình độ chuyên môn cao và kinh nghiệm nghề nghiệp thì mới có thể cung cấp những tư vấn tốt nhất cho sinh viên (Trần Thị Minh Đức \& Kiều Anh Tuấn, 2012). Đội ngũ CVHT phải đáp ứng yêu cầu về kiến thức chuyên môn, am hiểu những lĩnh vực liên quan đến lĩnh vực chuyên môn, ngành nghề và CTĐT, định hướng đầu ra, yêu cầu tuyển dụng, phương pháp học tập - nghiên cứu, các quy định - quy chế - chính sách. Ngoài ra, CVHT cần có kỹ năng giao tiếp tốt, phẩm chất đạo đức tốt, có trách nhiệm, nhiệt tình và hết lòng với sinh viên (Nguyễn Thị Út Sáu, 2013).

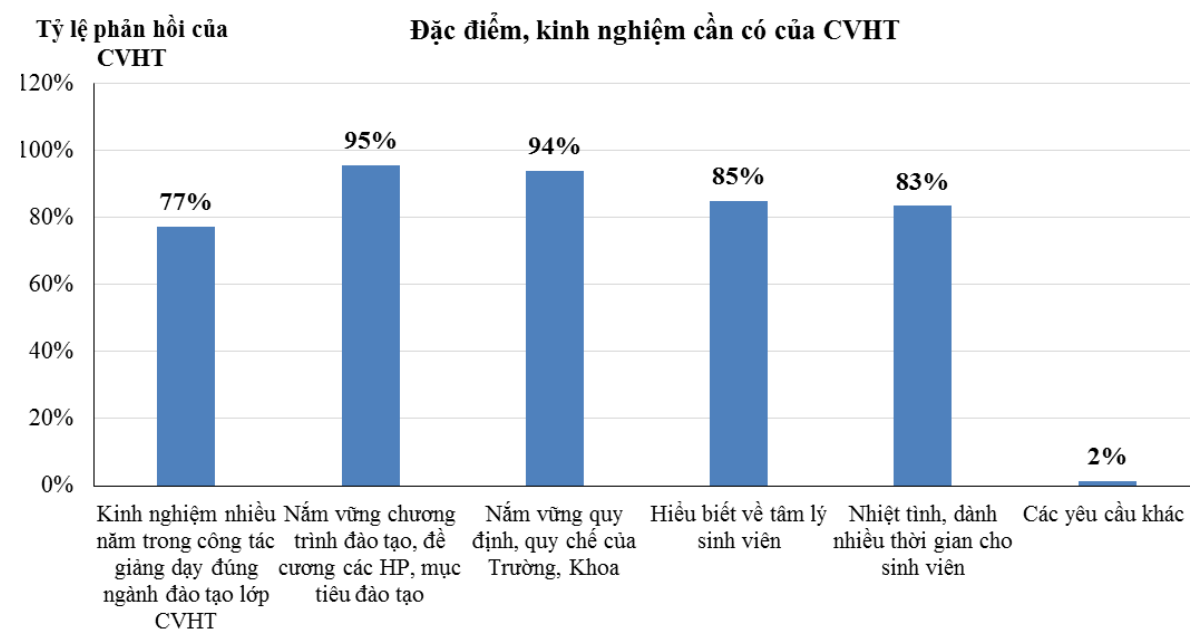

Hình 1. Đặc điểm, năng lực và kinh nghiệm cần có của CVHT

Kết quả khảo sát ý kiến của giảng viên đang đảm nhiệm công tác CVHT tại Khoa Công Nghệ cho thấy các đặc điểm, kinh nghiệm, yêu cầu cần thiết mà CVHT cần phải có là: (1) nắm vững mục tiêu đào tạo, nội dung CTĐT, đề cương chi tiết học phần (95\%); (2) nắm vững quy định, quy chế của Trường, Khoa (94\%); (3) hiểu biết về tâm lý sinh viên để có thể tư vấn cho sinh viên khi cần thiết $(85 \%)$; (4) nhiệt tình, dành thời gian để lắng nghe và chia sẻ với sinh viên $(83 \%)$; $(5)$ có nhiều năm kinh nghiệm trong công tác giảng dạy và nghiên cứu khoa học đúng ngành học của lớp CVHT (77\%). Các kết quả này đều cho thấy sự tương thích với các nghiên cứu đã được công bố trước đây. Ngoài 5 yếu tố trên, kết quả phản hồi cho thấy CVHT cũng cần có những đặc điểm năng lực khác như: có kỹ năng mềm, có hiểu biết về các vấn đề xã hội, có kinh nghiệm liên quan đến tuyển dụng việc làm, với tỷ lệ đề xuất thấp ( $2 \%$ ). Đối với giảng viên thực hiện công tác CVHT 
lần đầu tiên, việc thực hiện nhiệm vụ CVHT có nhiều khó khăn do chưa nắm rõ hết các quy định, quy chế của Trường.

\subsection{Các nội dung cần sinh hoạt cho sinh viên trong buổi họp CVHT}

CVHT có hai nhiệm vụ và chức năng cơ bản là quản lý sinh viên và tư vấn học tập cho sinh viên. Trong đó, nội dung tư vấn học tập cho sinh viên là vấn đề khá phức tạp và bao gồm nhiều nội dung. Các nội dung tư vấn học tập cho sinh viên có thể phân làm 4 nhóm chính: (1) CTĐT và xây dựng kế hoạch học tập, (2) phương pháp học tập theo học chế tín chỉ, (3) hoạt động nghiên cứu khoa học và (4) tư vấn huớng nghiệp và việc làm (Nguyễn Thị Út Sáu, 2013). Để hoạt động tư vấn của CVHT có hiệu quả, công tác CVHT và tư vấn phải được thực hiện đúng lúc, kịp thời, phù hợp với đối tượng và nhóm đối tượng, đa dạng và linh hoạt. (Nguyễn Thị Út Sáu, 2013; Trường Đại học Cần Thơ, 2011).

Khi được hỏi về những nội dung nào mà thường được CVHT tư vấn, sinh hoạt cho sinh viên, kết quả phản hồi của 66 CVHT cho thấy có 5 nhóm thông tin được CVHT truyền đạt đến sinh viên trong các buổi họp với tần suất cao trên $80 \%$ là: (1) tư vấn chuyên môn về ngành học $(97 \%),(2)$ các nội dung thông báo của Trường, Khoa ở từng thời điểm (89\%); (3) giới thiệu và tư vấn về kế hoạch học tập toàn khóa, phương pháp học tập $(88 \%)$; (4) định hướng nghề nghiệp (85\%); (5) giới thiệu, giải thích về nội dung CTĐT (83\%). Đây chính là 5 nội dung được sinh viên quan tâm và được tư vấn trong các cuộc họp CVHT và qua các kênh thông tin khác. Các nội dung này được đánh giá là rất hữu ích cho sinh viên trong quá trình học tập và rèn luyện tại Trường. Bên cạnh đó, các nội dung liên quan đến chính sách học bổng cho sinh viên, đánh giá và tư vấn các vấn đề liên quan đánh giá rèn luyện, điểm rèn luyện cũng được CVHT quan tâm nhắc nhở, tư vấn cho sinh viên nhưng với tần suất thấp hơn là $74 \%$ và $67 \%$. Các nội dung liên quan đến cuộc sống đời thường, sinh hoạt xã hội, nề nếp ký túc xá,... được CVHT quan tâm tư vấn cho sinh viên với tỷ lệ 59\%. Kế tiếp, nội dung tư vấn liên quan đến công tác nghiên cứu khoa học của sinh viên chiếm tỷ lệ phản hồi $55 \%$; liên quan đến giải quyết khiếu nại cho sinh viên là $52 \%$; phổ biến về các thủ tục hành chánh, chế độ chính sách khác của Trường, Khoa, Bộ môn là 39\%. Ngoài ra, các nội dung liên quan đến rèn luyện chính trị, đạo đức, tư tưởng lối sống, thái độ học tập và rèn luyện của sinh viên cũng được CVHT lồng ghép vào trong những buổi sinh hoạt thông qua các nội dung trên đây. Bên cạnh việc sinh hoạt của CVHT, Bộ phận văn phòng Khoa cũng thực hiện công tác thông tin đến sinh viên những nội dung liên quan đến thông báo, nội quy, quy định mới của Trường, Khoa một cách kịp thời, nhanh nhất.

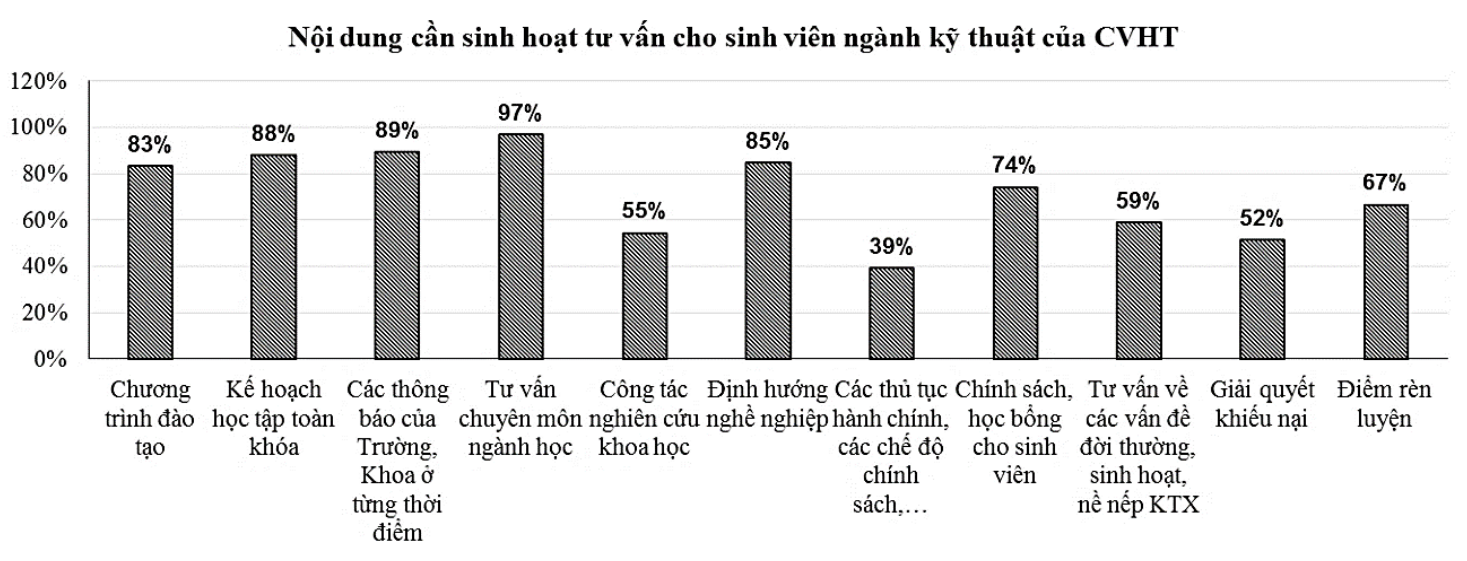

Hình 2. Những nội dung cần sinh hoạt cho sinh viên ngành kỹ thuật của CVHT

\subsection{Các phương thức trao đổi thông tin}

\subsubsection{Các kênh giao tiếp giũa CVHT - sinh vien}

Nếu như trước đây, hình thức liên lạc giữa CVHT với sinh viên được đánh giá ở mức thường xuyên là: gặp trực tiếp sinh viên, họp lớp và gọi điện thoại (Trần Thị Minh Đức \& Kiều Anh Tuấn, 2012), thì đến nay các kênh giao tiếp đã đa dạng hơn rất nhiều. Nhờ vào sự phát triển mạnh mẽ của công nghệ thông tin, hệ thống hỗ trợ công tác CVHT trên điện thoại di động cũng đã được nghiên cứu triển khai, giúp cho CVHT có thể quản lý, truy xuất thông tin của sinh viên nhanh chóng và thuận lợi, nâng cao hiệu quả công tác CVHT (Trần Công Án và ctv., 2016). Ngoài lịch họp lớp trực tiếp giữa CVHT với 
sinh viên theo kế hoạch thời khóa biểu được bố trí, CVHT còn sử dụng nhiều kênh thông tin khác nhau để liên lạc với sinh viên.

Theo kết quả khảo sát giảng viên tại Khoa Công Nghệ, có $88 \%$ CVHT sử dụng hệ thống email của Trường Đại học Cần Thơ để trao đổi, liên lạc thông tin với sinh viên. Tất cả các sinh viên của Đại học Cần Thơ đều được Trường cung cấp một tài khoản email, đây là kênh thông tin chính thống được sử dụng trong việc trao đổi học thuật, liên lạc thông tin, thông báo của Trường. Song song đó, có $71 \%$ CVHT sử dụng điện thoại để liên lạc, $56 \%$ CVHT dùng mạng xã hội "zalo group" để thông tin, 45\% sử dụng mạng xã hội tạo nhóm trên Facebook. Theo kết quả khảo sát này, một CVHT có thể sử dụng đồng thời một vài phương tiện trong giao tiếp với sinh viên. Việc tạo nhóm trên mạng xã hội như zalo hoặc facebook đa phần thuộc về các CVHT trẻ, kết quả cho thấy tính hiệu quả cao trong quá trình chuyển tải các thông tin giao tiếp qua lại giữa $\mathrm{CVHT}$ đến sinh viên, cũng như công tác quản lý lớp CVHT bằng phương thức này. Trong khi đó, CVHT có độ tuổi từ 50 trở lên, ít khi sử dụng mạng xã hội để trao đổi thông tin với sinh viên; việc trao đổi chủ yếu dựa trên các kênh email, điện thoại.

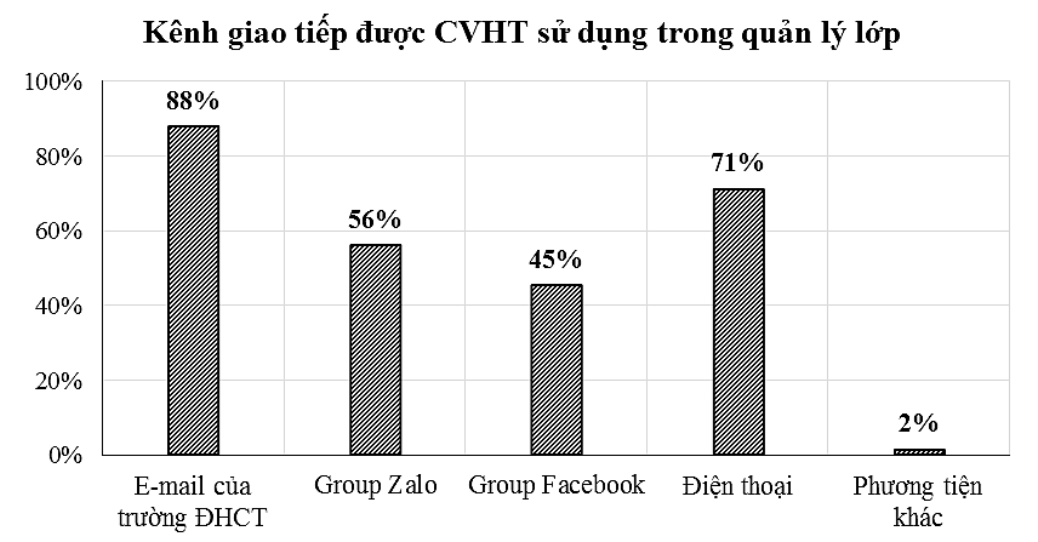

\section{Hình 3. Những kênh giao tiếp được CVHT sử dụng trong quản lý và giao tiếp với sinh viên}

Khi được hỏi kênh thông tin nào hiệu quả nhất trong công việc giao tiếp và triển khai thông tin đến sinh viên, phản hồi của giảng viên đang làm nhiệm vụ CVHT cho thấy kênh nhóm Zalo là hiệu quả nhất, kế đến là kênh thư điện tử email của Trường Đại học Cần Thơ, nhóm facebook và sau cùng là sử dụng điện thoại trực tiếp (Hình 4). Kết quả này cho thấy việc sử dụng các mạng xã hội trong công tác CVHT ngày càng thịnh hành so với điện thoại trực tiếp như trước đây, nhờ vào sự đáp ứng nhanh, chính xác, nhiều người tham gia cùng lúc, đặc biệt là ít tốn chi phí.

\section{Kênh thông tin hiệu quả nhất được CVHT sử dụng}

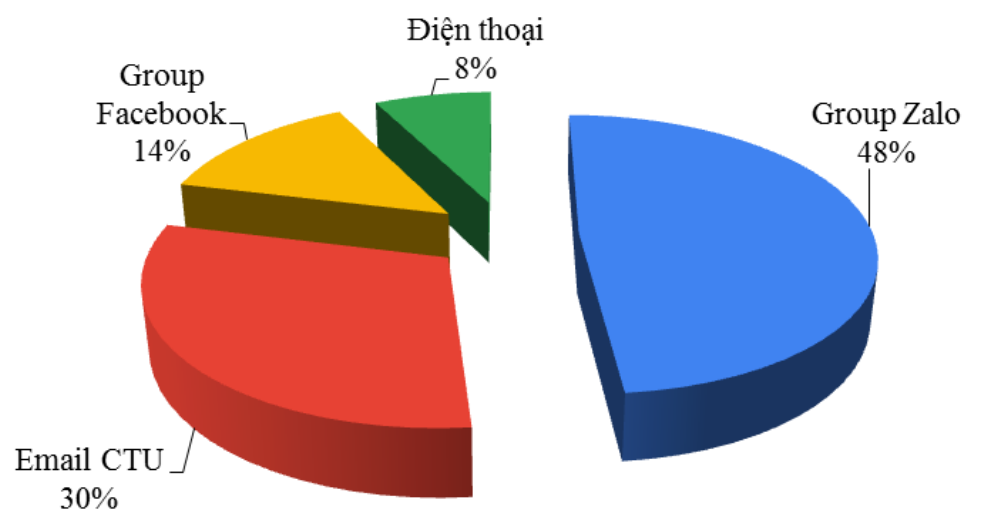

Hình 4. Những kênh thông tin hiệu quả nhất được CVHT sử dụng 


\subsubsection{Thời luợng sinh hoạt CVHT trên lớp}

Hiện tại, tại Trường Đại học Cần Thơ đã sắp xếp thời khóa biểu chính thức để CVHT sinh hoạt với sinh viên, trung bình 4 buổi/học kỳ, thời lượng 50 phút/buổi sinh hoạt. Thời lượng này có thể không đủ để CVHT trao đổi thông tin với sinh viên năm thứ nhất, do cần phải tư vấn rất nhiều vấn đề liên quan đến việc xây dựng kế hoạch học tập toàn khóa, CTĐT, phương pháp học tập, và các vấn đề khác để tân sinh viên hòa nhập tốt với cuộc sống giảng đường đại học. Đối với sinh viên các năm kế tiếp, thời lượng này đảm bảo đủ để sinh hoạt các nội dung liên quan. Tuy nhiên, với sinh viên năm cuối đang cần rất nhiều sự tư vấn của CVHT về LVTN, cơ hội việc làm, định hướng nghề nghiệp sau khi ra trường, và nhiều thông tin khác; thì thời lượng 50 phút/buồi đôi khi chưa đủ đáp ứng. Bên cạnh đó, đôi khi CVHT cần giải quyết trả lời thắc mắc của sinh viên ngay lúc cần thiết, không nhất thiết phải chờ đến buổi họp CVHT, nên thời lượng làm việc với sinh viên của CVHT nhìn chung là nhiều hơn so với thời lượng đã được bố trí theo kế hoạch thời khóa biểu. Theo kết quả khảo sát tại Khoa Công Nghệ, có ý kiến CVHT cho rằng việc bố trí lịch họp với sinh viên là chưa phù hợp, có những buổi họp chưa có nội dung quan trọng để chia sẻ, vì các nội dung đã được $\mathrm{CVHT}$ tư vấn và trả lời cho sinh viên trước đó. Tuy nhiên, việc duy trì các buổi họp CVHT theo thời khóa biểu sẽ đạt được các mục tiêu khác như: sự quan tâm của nhà trường đến sinh viên, sự quan tâm của giảng viên đến sinh viên, đặc biệt là sự đoàn kết gắn bó giữa các sinh viên trong lớp học - điều này sẽ thúc đẩy quá trình học tập, rèn luyện, phấn đấu của sinh viên một cách tốt hơn. Ngoài ra, nếu như không có thời khóa biểu sinh hoạt CVHT chính thức, việc tổ chức họp lớp/nhóm trong học kỳ có sự chênh lệch và không đồng nhất giữa CVHT (Đặng Thị Ngọc Lan, 2015). Các CVHT đề xuất xem xét tăng thời lượng sinh hoạt CVHT đối với sinh viên năm thứ nhất, vì có nhiều vấn đề liên quan cần phải hướng dẫn cho sinh viên trong giai đoạn này.

\subsubsection{Tần suất sinh viên cần được tu vấn tù CVHT}

Tần suất sinh viên cần được tư vấn trong khoảng 4 tuần thường từ 1 đến 5 câu hỏi/lần họp được đặt ra cho CVHT, chiếm tỷ lệ là $82 \%$ (54/66 CVHT được khảo sát). Điều này hoàn toàn hợp lý đối với hệ thống quản lý CVHT hiện nay của Khoa Công nghệ - Trường Đại học Cần Thơ. Trong thời gian học, ngoài việc được tư vấn bởi CVHT, sinh viên còn được tư vấn bởi giảng viên, Bộ môn, Ban chủ nhiệm Khoa bất kỳ lúc nào cần thiết, khi mà các thắc mắc của sinh viên không được CVHT giải quyết thỏa đáng; không nhất thiết phải đợi đến buổi họp với CVHT. Bên cạnh đó, số nhu cầu cần tư vấn của sinh viên nhiều hơn 5 câu hỏi/lần họp xảy ra ở tần suất thấp hơn, thuộc vào nhóm sinh viên năm thứ 1 và năm cuối, số câu hỏi này cũng phụ thuộc vào sự nhiệt tình, gần gũi của CVHT. Điều này cho thấy đối với sinh viên năm 1 và năm cuối cần có nhu cầu tư vấn nhiều hơn.

Ngoài các kết quả trên, thông tin phản hồi cho thấy $91 \%$ CVHT quan tâm, theo dõi và nắm rõ kết quả học tập của sinh viên; $18 \%$ CVHT theo dõi và biết được hoàn cảnh, điều kiện gia đình của sinh viên lớp CVHT phụ trách; $5 \%$ biết được điều kiện hoàn cảnh, điều kiện gia đình đối với một số sinh viên đặc biệt. Kết quả này cho thấy rằng $\mathrm{CVHT}$ chỉ quan tâm đến hoạt động học tập của sinh viên tại Trường, chưa quan tâm nhiều đến hoàn cảnh, điều kiện gia đình của sinh viên để có thể nắm bắt được tâm lý sinh viên cũng như hỗ trợ, tư vấn kịp thời trong quá trình học.

\subsection{Thuận lợi - khó khăn trong công tác CVHT}

Kết quả khảo sát ý kiến của CVHT tại Khoa Công Nghệ cho thấy công tác CVHT có những thuận lợi và những khó khăn nhất định. Các kết quả phân tích này chỉ dựa trên các ý kiến phản hồi của giảng viên, chưa đề cập đến kết quả phân tích ý kiến phản hồi từ phía sinh viên.

\subsubsection{Thuận lợi}

Kết quả phản hồi từ CVHT cho thấy có 4 yếu tố thuận lợi trong công tác quản lý và tư vấn sinh viên của CVHT. Trong đó, yếu tố thuận lợi nhất vời $82 \%$ CVHT chọn đó là lịch họp cụ thể theo thời khóa biểu; điều này cũng tương ứng với ý kiến được $59 \%$ lựa chọn, đó là sinh viên tham gia họp CVHT như lịch học của thời khóa biểu (Hình 5). Kết quả này cho thấy rằng mức độ hài lòng của giảng viên về kế hoạch tổ chức họp CVHT được hoạch định rõ ràng là hết sức quan trọng, giúp cho giảng viên và sinh viên sắp xếp thời gian và tuân thủ theo kế hoạch chung một các hiệu quả nhất. Yếu tố thuận lợi kế tiếp sự phân bố thời lượng hợp lý và quy định về công tác CVHT rõ ràng với cùng tỷ lệ phản hồi là $45 \%$. Ngoài các nội dung trên, một số ít CVHT (3\%) cho rằng ứng dụng các mạng xã hội để liên lạc nhanh với sinh viên là một thuận lợi trong công tác $\mathrm{CVHT}$ hiện nay; nhờ đó, CVHT có thể tư vấn thường xuyên và kịp thời cho sinh viên ngay khi có sự việc cần thiết. 
Tỷ lệ phản hồi của CVHT về những thuận lợi trong công tác

CVHT với ngành kỹ thuật

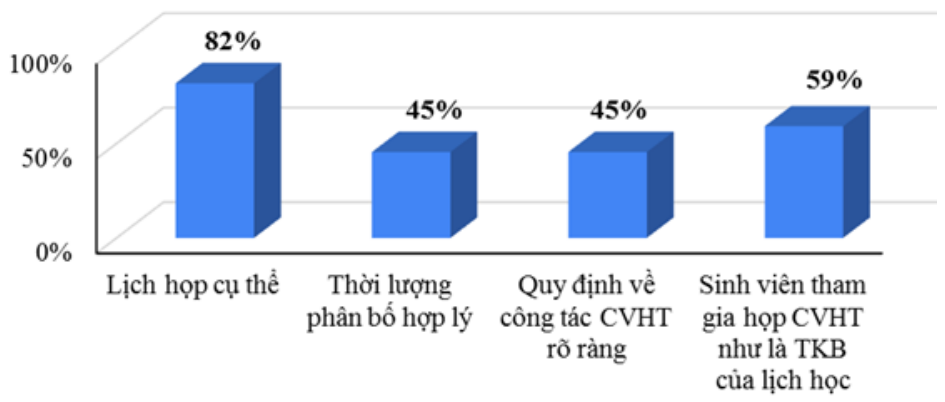

Hình 5. Những thuận lợi chính trong công tác CVHT với các ngành kỹ thuật

Kết quả nghiên cứu tại Khoa Công Nghệ Trường Đại học Cần Thơ cho thấy có một sự khác biệt với kết quả được công bố trước đây của Trần Thị Minh Đức (2012). Theo nghiên cứu này, sự giúp đỡ của ban cán sự lớp, sự nhiệt tình của sinh viên, sự khích lệ quan tâm của lãnh đạo, sự đam mê công việc của CVHT là 4 yếu tố thuận lợi trong công tác CVHT (Trần Thị Minh Đức \& Kiều Anh Tuấn, 2012). Sự khác biệt này cho thấy hiện tại các yếu tố thuận lợi của công tác CVHT tại Khoa Công Nghệ liên quan đến sự tổ chức khoa học, nội dung quy định rõ ràng; giảng viên thực hiện công tác CVHT như là một nhiệm vụ của mình; sinh viên nhận thức được sự quan trọng và hữu ích khi được CVHT tư vấn và hỗ trợ.

\subsubsection{Khó khăn}

Bên cạnh những thuận lợi đã được trình bày trên đây, công tác CVHT vẫn còn tồn tại những khó khăn nhất định cần phải được khắc phục. Kết quả nghiên cứu này cho thấy có 8 yếu tố khó khăn ảnh hưởng đến công tác CVHT ở khối ngành kỹ thuật thuộc Khoa Công Nghệ như thể hiện ở Hình 6. Trong đó, sinh viên không tham gia họp lớp với CVHT theo thời khóa biểu (TKB) chiếm tỷ lệ phản hồi cao nhất (55\%); mặc dù số lượng sinh viên này là không nhiều và tập trung vào các sinh viên cá biệt, cần được tư vấn hỗ trợ. Kế tiếp, yếu tố về thời gian của giảng viên, tài liệu nội dung phổ biến trong sinh hoạt CVHT, giảng viên chưa nắm rõ quy định của Trường (Khoa) là những khó khăn có tỷ lệ phản hồi khá cao $38 \%, 33 \%$ và $29 \%$.

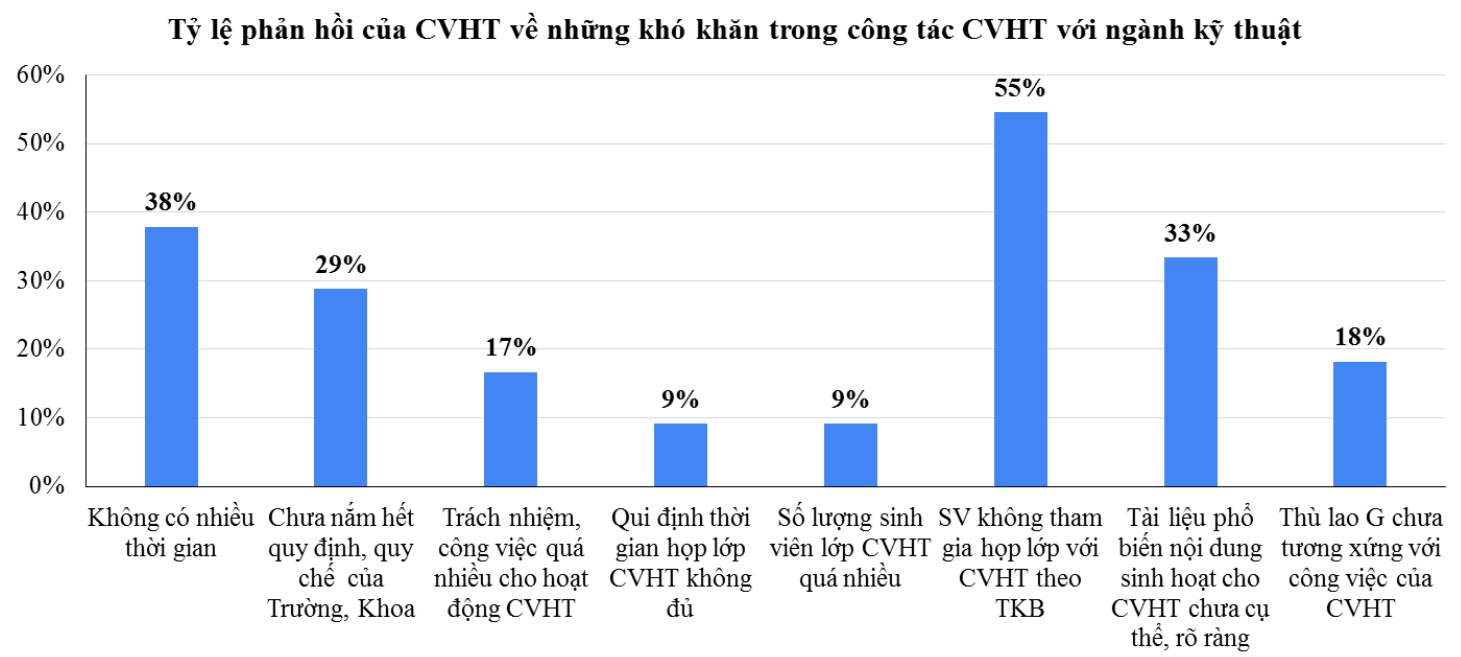

Hình 6. Những khó khăn chính trong công tác CVHT với các ngành kỹ thuật 
Bên cạnh đó, yếu tố về trách nhiệm và công việc quá nhiều được phân công cho CVHT, thù lao chưa tương xứng với công việc cũng được một số giảng viên đang làm nhiệm vụ CVHT đề cập với tỷ lệ là $17 \%$ và $18 \%$. Sau cùng, yếu tố thời gian họp không đủ, số lượng sinh viên đông cũng được các CVHT phản hồi với tỷ lệ thấp $(9 \%)$. Ngoài ra, một số ít ý kiến CVHT cho rằng một số sinh viên còn không tập trung trong giờ họp, không xem trọng việc lập KHHT, chưa năng động trong việc tìm hiểu các quy định, quy chế của Trường/Khoa; CVHT không có đủ chuyên môn về tâm lý học để tư vấn cho sinh viên. Nếu so sánh với những nghiên cứu trước đây, số sinh viên lớp CVHT quá đông, thù lao cho CVHT chưa hợp lý là 2 khó khăn đã được chỉ ra (Trần Thị Minh Đức \& Kiều Anh Tuấn, 2012).

\subsection{Tăng cường hiệu quả công tác CVHT}

Kết quả nghiên cứu qua khảo sát giảng viên đang thực hiện nhiệm vụ CVHT tại Khoa Công Nghệ Trường Đại học Cần Thơ cho thấy rằng: hầu hết các CVHT đều thực hiện và hoàn thành tốt công tác CVHT. Một số ít CVHT chưa có đủ kinh nghiệm trong công tác giảng dạy, công tác chuyên môn; chưa nắm được vững vàng nội dung các quy định, quy chế của Trường/Khoa; chưa nắm được rõ cụ thể nội dung sinh hoạt CVHT, nên sinh viên còn có những thắc mắc cần được các bộ phận chuyên trách giải đáp. Để tăng cường công tác CVHT với các ngành kỹ thuật một cách khoa học, hiệu quả, một số đề xuất cần phải được thực hiện như sau:

(1)Cần phải xây dựng đội ngũ CVHT nhiệt tình, năng động, có kinh nghiệm trong giảng dạy, am hiểu chuyên môn ngành nghề, nắm vững chương trình đào tạo, nội dung các học phần, nắm vững các quy định của Trường/Khoa để có thể tư vấn và định hướng cho sinh viên.

(2)Cần phải xây dựng được sổ tay CVHT, trong đó quy định các "nội dung sinh hoạt cứng" cho sinh viên tương ứng với từng năm, từng học kỳ để CVHT dựa vào đó triển khai, bổ sung những "nội dung mềm" tùy thuộc vào điều kiện và tình hình thực tế xã hội, ngành nghề tương ứng.

(3)Cần phải có các buổi tập huấn chuyên môn về công tác CVHT cho các giảng viên đang thực hiện nhiệm vụ CVHT, để bồi dưỡng và nâng cao năng lực quản lý lớp, trao đổi kinh nghiệm, hiểu biết về quy định quy chế của Trường/Khoa.

(4)Cần phải duy trì kế hoạch sinh hoạt lớp CVHT theo thời khóa biểu để sinh viên, giảng viên sắp xếp bố trí thời gian thực hiện đúng theo kế hoạch.

(5)Cần sử dụng các hình thức, phương tiện, công nghệ thông tin trong giao tiếp để tăng cường công tác CVHT ngày càng hiệu quả hơn, ngoài việc họp mặt trực tiếp theo thời khóa biểu.

\section{KẾT LUẬN}

Công tác CVHT có một ý nghĩa rất quan trọng trong hệ thống đào tạo tín chỉ. Giảng viên thực hiện công tác CVHT như một vai trò cầu nối không thể thiếu được trong mối quan hệ nhà trường - sinh viên - thị trường lao động; hỗ trợ và tư vấn cho sinh viên rất nhiều vấn đề liên quan trong suốt quá trình học tập tại Trường. Kết quả nghiên cứu này cho thấy được những đặc điểm năng lực của một $\mathrm{CVHT}$ cần có, những thuận lợi và khó khăn khi triển khai thực hiện công tác CVHT đối với các ngành kỹ thuật. Các giải pháp đề xuất để tăng cường công tác CVHT không chỉ áp dụng cho ngành kỹ thuật, mà còn có thể triển khai áp dụng cho quy mô rộng hơn với các ngành nghề khác nhau, để công tác CVHT đạt được hiệu quả tốt nhất.

\section{LÒ̀I CẢM ƠN}

Tác giả xin chân thành cảm ơn quý giảng viên đang thực hiện nhiệm vụ CVHT các lớp thuộc Khoa Công Nghệ - Trường Đại học Cần Thơ đã nhiệt tình tham gia khảo sát, phản hồi, đóng góp ý kiến cho nghiên cứu này.

\section{TÀI LIỆU THAM KHẢO}

Đặng Thị Ngọc Lan. (2015). Thực trạng công tác cố vấn học tập và rèn luyện của đội ngũ cố vấn học tập ở Trường Đại học Sư phạm Kỹ thuật TP HCM. Tap chi khoa hoc ĐHSP TPHCM, 6(72), 123-134.

Nguyễn Nguyệt Minh. (2015). Nâng cao vai trò của cố vấn học tập trong các trường Cao Đẳng theo học chế tín chỉ. Tạp chi Giáo duc, 366, 5-6.

Nguyễn Thị Ứt Sáu. (2013). Một số vấn đề lý luận về hoạt động tư vấn học tập cho sinh viên của cố vấn học tập ở các trường đại học. Tạp chi Giáo $d u c, 318(2), 17-19$

Trần Công Án, Lâm Chí Nguyện, Đoàn Hòa Minh, Phan Tấn Tài, Phạm Hữu Tài, Châu Xuân Phương, \& Sơn Búp Pha. (2016). Hệ thống hỗ trợ cố vấn học tập trên thiết bị di động. Tạp chí khoa học Đại học Cần Thơ, 47, 47-58.

Trần Thị Minh Đức, \& Kiều Anh Tuấn. (2012). Cố vấn học tập trong các trường Đại học. Tạp chí Khoa học ĐHQGHN, Khoa học Xã hội và Nhân văn, 28, 23-32. 
Trường Đại học Cần Thơ. (2011). Kỷ yếu Hội nghị nâng cao vai trò của Cố vấn học tập. Trường Đại học Cần Thơ, 72 trang.

http://cdsonla.edu.vn/daotao/attachments/article/ 451/CVHT.pdf.

Trường Đại học Cần Thơ. (2020a). Quyết định ban hành quy định về công tác học vụ dành cho sinh viên trình độ đại học hệ chính quy của Trường Đại học Cần Thơ (Số 2093/QĐ-ĐHCT).

https://dsa.ctu.edu.vn/images/upload/vbanply/20
20/Quychehocvu/QD2093_Quy-dinh-cong-tachoc-vu_2020.pdf.

Trường Đại học Cần Thơ. (2020b). Quyết định ban hành quy định về công tác cố vấn học tập tại Trường Đại học Cần Thơ (Số 3873/QĐ-ĐHCT). https://dsa.ctu.edu.vn/images/upload/vbanply/20 20/CVHT/03_3873KHTH_10-11-2020.pdf. 